\title{
Face recognition method based on gabor wavelet and memetic ecological algorithm.
}

\section{Shoujun Tang*}

The Open University of Guangdong, Guangdong Polytechnic Institute, Guangzhou, Guangdong 510091, PR China

\begin{abstract}
At present, with the development of science and technology, a lot of new technologies have been developed, which has brought great convenience to people's lives. Face recognition technology is a very innovative technology. Therefore, in the field of computer science, professionals have been studying the related technologies. And the technology has been applied in the fields of industry and biology. In this paper, a deep analysis of the theory of face recognition was made. Through the gabor wavelet and memetic ecological algorithm, the technology was further studied.
\end{abstract}

Keywords: Multimedia, Computer technology, Employment guidance.

\section{Introduction}

One of the most important research areas in the field of biological identification is the technology of face recognition. The technology includes the field of image processing, pattern recognition and visual recognition technology. Therefore, the use of the technology is very broad, and many people from all walks of life are very interested in this [1]. Many other techniques in the field of biology, such as the identification of human fingerprints, and the identification of the human eye, are less likely to be accepted. Face recognition technology is relatively simple, and has been praised by the majority of users [2]. In the application, the face recognition technology does not need the user's cooperation. Therefore, in the application, it will be more convenient and quick.

\section{State of the Art}

For a variety of light, gesture and other factors, Gabor wavelet can have good recognition. Therefore, in the face recognition,
Gabor wavelet is used frequently, and has become a very important technology. The technique can easily collect various features of human face, and it is very easy to recognize and describe all kinds of information. Memetic algorithm is a widely used algorithm in the face recognition technology, which can locate and transform the face information, and can quickly match the original image.

\section{Methodology}

The Gabor wavelet technology can effectively identify the edge of the identified region image, and can measure the measurement direction, area and scale. And when the technology is used, it will not be interfered by the light. Therefore, it can be used at any time and any place, in order to well understand all kinds of information. These advantages make the technology widely used in the field of face recognition.

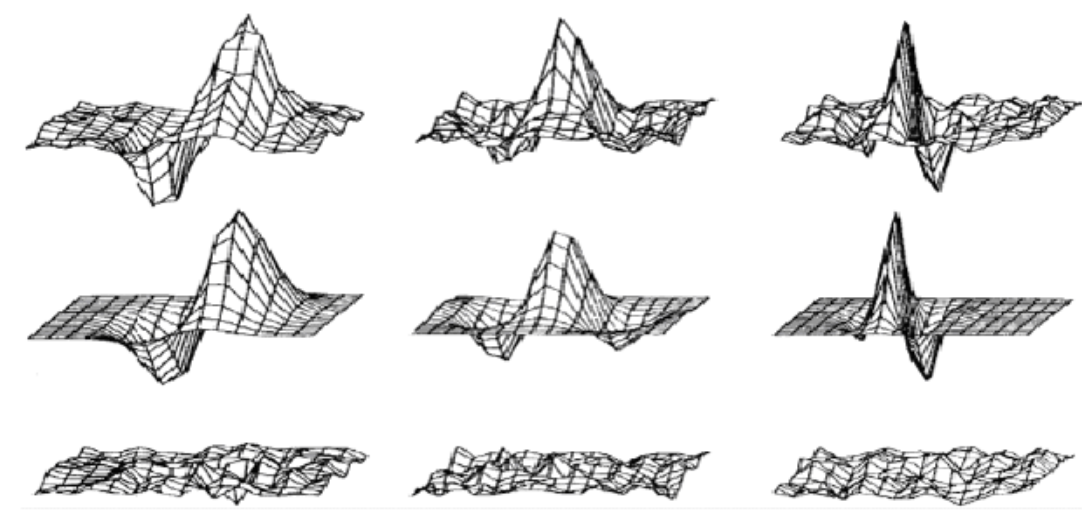

Figure 1. The response of Gabor wavelet to biological cortex. 
When applied in the field of face recognition, Gabor wavelet technology has the following advantages. Firstly, the Gabor wavelet technology can be used to make the response to the characteristics of the images with different angles, area and size, and can grasp the feature points above the image accurately (Figure 1). In the Gabor wavelet technology, the larger scale band can ignore the noise of the image to a certain extent, so as to prevent interference in recognition. However, in the Gabor wavelet technology, the small band can be used to identify the fine features of the image, the details and so on. However, this band is relatively easy to be disturbed by the noise with the image itself.

In the process, the Gabor wavelet can be very good to grab the key information of the local, and analyze the characteristics of the frequency of information. In this paper, the identification model of Gabor wavelet in face recognition technology is studied.

Firstly, when the band frequency of the Gabor wavelet is used to process the information, the information will be set constant. For example, $\mathrm{x}, \mathrm{y}, \mathrm{z}$, these constants are set as a coordinate axis. Thus, the whole direction and size of the identified image are confirmed. Therefore, when the spatial coordinates of the image points are confirmed, the image can be better analysed and judged.

Secondly, the information should be calculated by the following formula:

$\mathrm{K}_{\mathrm{V}}=\mathrm{K}_{\text {max }} / \mathrm{fV}$ (1)

Then, the results of the analysis are standardized to $v=\{1,2,3$, and 4 \}. Finally, the calculation results are analyzed.

\section{Result Analysis and Discussion}

When applied to the field of real life recognition, Gabor wavelet technology can eliminate the DC component directly by the algorithm, and can resist the interference caused by the change of different light. Therefore, in the situation of complex light, the technology will usually be used for face recognition. First of all, 10 testers are chosen as the research objects to carry out the face recognition. The following table is captured face data (Table 1).

Table 1. Data of face recognition technology.

\begin{tabular}{llllll}
\hline Item & 1 & 2 & 3 & 4 & 5 \\
\hline $\begin{array}{l}\text { Recognition } \\
\text { resolution }\end{array}$ & 89.55 & 89.24 & 96.25 & 63.45 & 40.23 \\
\hline $\begin{array}{l}\text { Recognition } \\
\text { accuracy }\end{array}$ & 77.34 & 64.35 & 89.24 & 46.35 & 49.55 \\
\hline Recognition speed & 99.63 & 74.34 & 68.46 & 83.23 & 88.67 \\
\hline
\end{tabular}

After the above analysis, it is found that when applied to various fields, face recognition technology can identify the identity of people entering the designated area in a very short time. For those who do not meet the requirements of the identity, it can quickly identify and refuse these personnel, thereby ensuring the security and confidentiality of the region. The following Figure 2 shows the process of face scanning.
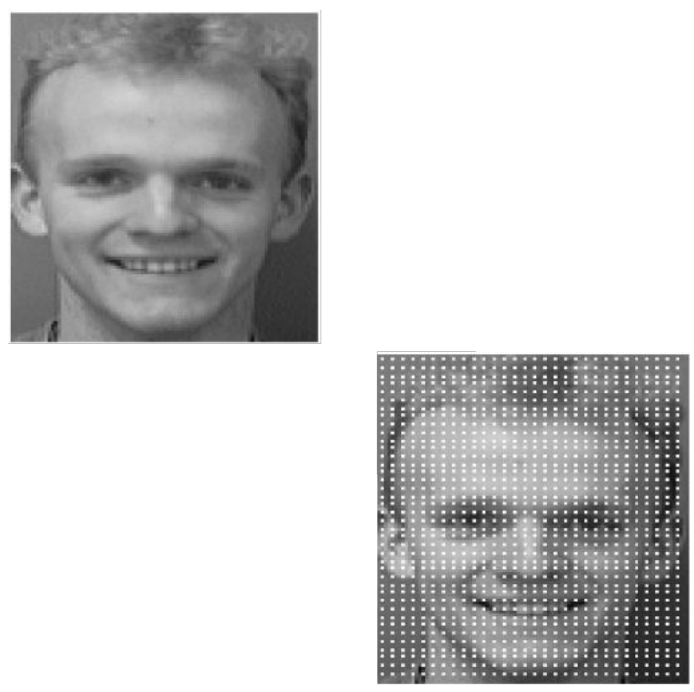

Figure 2. The scanning process of face.

The application effect of Gabor wavelet technology in face recognition is obvious. Through the above data we can see that in the identification of clarity, recognition accuracy and recognition speed, Gabor wavelet technology has very important advantages. There is a very bright prospect for the face recognition technology.

\section{Conclusions}

Due to the current flood of information from all walks of life, information or regional protection and confidentiality have received more and more attention. People hope to realize information security through a variety of new technologies. In the rapid development of science and technology today, people can use a lot of technologies, and the face recognition technology is a very important science and technology development. Based on the analysis of the theory of Gabor wavelet and Memetic ecological algorithm, some research objects were selected, and the effect of these technologies was studied and evaluated, thus greatly helping the development of face recognition technology.

\section{References}

1. Schroff F, Kalenichenko D, Philbin J. Facenet: A unified embedding for face recognition and clustering. Proceed IEEE Conf on Comp Vis and Patter Recog 2015; 815-823.

2. Parkhi OM, Vedaldi A, Zisserman A. Deep Face Recognition, BMVC 2015; 1: 6.

\section{*Correspondence to}

Tang Shoujun

The Open University of Guangdong

Guangdong Polytechnic Institute

PR China 